\title{
Comparison Between Selective Caudal Fixed Screw Construct and All Variable Screw Construct in Anterior Cervical Discectomy and Fusion
}

\section{Jae Jun Yang}

Dongguk University Ilsan Hospital

Sehan Park ( $\sim$ birdone86@gmail.com )

Dongguk University Ilsan Hospital

\section{Seongyun Park}

Dongguk University Ilsan Hospital

\section{Research Article}

Keywords: Anterior cervical discectomy and fusion, dynamic plating, fixed screw, variable screw, adjacent level ossification development

Posted Date: December 29th, 2020

DOl: https://doi.org/10.21203/rs.3.rs-131415/v1

License: (a) (1) This work is licensed under a Creative Commons Attribution 4.0 International License. Read Full License 


\section{Abstract}

Objectives: This retrospective comparative study aimed to compare the efficacy of selective caudal fixed screw constructs with all variable screw constructs in anterior cervical discectomy and fusion (ACDF).

Methods: Thirty-five patients who underwent surgery using selective caudal fixed screw construct (SF group) were compared with 44 patients who underwent surgery using all variable constructs (AV group). The fusion rate, subsidence, adjacent level ossification development (ALOD), adjacent segmental disease (ASD), and plate-adjacent disc space distance were assessed.

Results: The one-year fusion rates assessed by computed tomography bone bridging and interspinous motion as well as the significant subsidence rate did not differ significantly between the AV and SF groups. The ALOD and ASD rates and plate-adjacent disc space distances did not significantly differ between the two groups at both the cranial and caudal adjacent levels. The number of operated levels was significantly associated with pseudarthrosis in the logistic regression analysis.

Conclusion: The stability provided by the locking mechanism of the fixed screw did not lead to an increased fusion rate at the caudal level. Therefore, the screw type should be selected based on individual patient's anatomy and surgeon's experience without concern for increased complications caused by screw type.

\section{Introduction}

Anterior cervical plating has been widely applied in anterior cervical discectomy and fusion (ACDF) to enhance fusion rate, improve cervical alignment, and prevent graft subsidence. ${ }^{1-5}$ However, the anterior cervical plate is not without implant-related complications, including screw migration and fracture. ${ }^{6-8}$ Furthermore, a plate placed proximal to the adjacent disc space reportedly increases the incidence of adjacent level ossification development (ALOD), which can adversely affect the range of motion and degeneration of unoperated levels. ${ }^{9-11}$ Therefore, plating techniques that can minimize the chance of complications while taking advantages are needed. ${ }^{10,12,13}$

Dynamic plating has been commonly used because it can avoid distraction force at the graft-bone interface and stress shielding. ${ }^{14}$ The locking and variable screws are two types of screws used for dynamic plating. Because the insertion angle is more freely adjustable with variable types, endplate injury can be avoided with a variable screw even when the screw insertion point is near the operated disc space. Therefore, a variable screw is advantageous when keeping the plate-adjacent disc space distance to > $5 \mathrm{~mm}$, which is needed to avoid ALOD. ${ }^{9-11}$ Locking screws provide additional stability due to the locking mechanism at the screw-plate interface. Whereas locking screws allow rigid fixation, variable screws allow toggling or rotational movement, which demonstrates the advantages of dynamic plating. ${ }^{14}$ 
Previous reports have demonstrated that ALOD more commonly occurs at the proximal adjacent level compared to the distal adjacent level. ${ }^{9,10}$ Furthermore, pseudarthrosis or implant failure most commonly occur at the caudal-most level. ${ }^{6,15,16}$ Therefore, we attempted a hybrid construct using fixed screws at the caudal-most instrumented level and variable screws at the cranial and middle levels to prevent ALOD at the proximal adjacent level while minimizing the pseudarthrosis rate at the caudal level. This selective fixed screw construct would maintain a cranial plate-disc space distance of $>5 \mathrm{~mm}$ to prevent ALOD and provide further stability at the caudal-most level by using the locking screw mechanism of fixed screw. We hypothesized that a selective fixed screw construct would result in a higher fusion rate and a similar rate of ALOD or adjacent segment degeneration (ASD) as the all variable constructs.

\section{Materials And Methods}

\section{Patient characteristics and study design}

The study protocol was approved by the institutional review board of our institute (Dongguk University Ilsan Hospital Institutional Review Board). All methods were carried out in accordance with relevant guidelines and regulations. Informed consent was waived due to the retrospective nature of the study. We retrospectively reviewed 101 patients who underwent ACDF with plate augmentation between September 2012 and March 2018 who met the inclusion/exclusion criteria. Inclusion criteria were: (1) patients who had surgery due to degenerative cervical myelopathy/radiculopathy; and (2) those who were followed-up for at least 2 years postoperatively. Exclusion criteria were: (1) patients who underwent surgery due to tumor, infection, or trauma; (2) and those who had previous cervical operation. All operations were performed by a single surgeon (JJY).

Patients who underwent surgery using all the variable screw non-constrained constructs were categorized as the all variable group (AV group). Patients who underwent surgery using the hybrid construct with fixed screws at the lowermost instrumented vertebra and variable screws at rest of the levels were categorized as the selective fixed group (SF group) (Fig. 1). In our institute, the all variable screw constructs were used for patients who had surgery before September 2017. Next, the selective fixed construct was used for comparison.

\section{Data collection}

Clinical characteristics and radiological data of patients were collected from medical chart reviews. The neck and arm pain visual analogue scale (VAS) and neck disability index (NDI) scores were recorded preoperatively and at the final follow-up.

Radiological measurements were performed twice by a spine fellowship-trained surgeon. Cervical lordosis was measured by the angle between the lines passing through the lower margin of $\mathrm{C} 2$ and $\mathrm{C} 6$ or C7 vertebrae. ${ }^{17}$ Fusion was assessed at 12 months postoperatively by two methods using dynamic lateral radiographs and computed tomography (CT) images using the following criteria: (1) interspinous motion (ISM) $<2$ mm on a $150 \%$ magnified flexion/extension lateral radiograph ${ }^{18}$; and (2) bone bridging 
formation on sagittal and coronal reconstructed CT images. ${ }^{19}$ Subsidence was assessed when the distance between the endplate of vertebral body and edge of the allograft changed more than $2 \mathrm{~mm}$ in CT performed at 2 days and 1 year, postoperatively. Adjacent segments with disc height changes or osteophyte formation at the 1-year follow-up CT were identified as ASD. ${ }^{20}$ Adjacent-level ossification development was assessed when anterior longitudinal ligament ossification crossing the adjacent disc space was identified. ${ }^{9}$ The ASD and ALOD were assessed both at proximal and distal adjacent levels. The plate-adjacent disc space distance was measured as the distance between the tips of the plate to the cephalad and caudal adjacent disc spaces on the immediate postoperative lateral radiograph of the cervical spine (Fig. 2). ${ }^{9,10}$

\section{Surgical technique}

The standard Smith-Robinson approach was used to expose the indicated levels. After complete discectomy, the cartilage material was removed using a ring curette. Care was taken to achieve complete box-shaped endplate preparation. A corticocancellous allograft (Cornerstone ASR, Medtronic, Minneapolis, MN, USA) with an appropriate size was inserted into the disc space. Local autogenous bone grafts were inserted into the remaining empty disc space. ${ }^{21}$

For the AV group, cranial and caudal screws were inserted at the anterior endplate corners and angled away from the endplate to use the shortest cervical plate (Atlantis, Medtronic, Minneapolis, MN, USA) and longest screw to keep the cranial and caudal ends of the plate as far away from the adjacent discs as possible. All screws were inserted using the variable angle type. In the SF group, cranial screws were inserted using the same technique as described for the AV group. The fixed screws were inserted at the caudal level and variable screws were used at the rest of the levels. The insertion angle of the fixed-angle screw was $12^{\circ}$ caudal. Screw length was determined based on preoperative CT measurements. The patients wore a neck collar for 6-12 weeks.

\section{Statistical analysis}

Categorical variables such as sex, diagnosis, smoking status, complications, fusion, subsidence. ALOD, and ASD were analyzed using the chi-square test. Continuous variables such as age, BMI, BMD, follow-up period, number of levels, $\mathrm{C} 2-\mathrm{C} 7$ lordosis, and plate-adjacent disc distance were analyzed using the Student's t-test. A logistic regression analysis was performed to identify factors associated with pseudarthrosis. Intraobserver agreements were assessed using the intraclass correlation coefficient (ICC) and Kappa coefficient. All data management and analyses were performed using SPSS version 21.0 software (SPSS, Inc., Chicago, IL, USA). P-values $<0.05$ were considered significant.

\section{Results}

Of the 101 patients reviewed, 79 met the inclusion criteria, and were included in the study. Forty-four patients were included in the AV group (mean age, $56.1 \pm 12.4$ years; 26 men [59.1\%]) and 35 patients were included in the SF group (mean age, $56.1 \pm 12.2$ years; 21 men [60.0\%]). The AV group involved more 
radiculopathy patients than the SF group $(p=0.02)$. In contrast, there were no baseline differences between the two groups. Number of levels operated was $1.6 \pm 0.7$ levels for the AV group and $1.7 \pm 0.7$ levels for the SF group $(p=0.75)$ (Table 1). One patient $(2.3 \%)$ in the AV group underwent reoperation due to surgical site infection. One patient $(2.9 \%)$ in the SF group underwent reoperation due to adjacent segment degeneration.

The kappa coefficient for intraobserver reliability was 0.828 for the assessment of fusion and 0.768 for the assessment of subsidence. The ICC for intraobserver reliability was 0.833 for the measurement of cervical lordosis and 0.817 for the measurement of plate-adjacent disc space distance.

\section{Radiographic and clinical results}

One-year fusion rates assessed by CT bone bridging (28 [63.6\%] vs. 20 [57.1\%], p = 0.64), and ISM (30 [68.1\%] vs. 26 [74.3\%]) did not differ significantly between the AV and SF groups. Furthermore, the significant subsidence rate did not differ significantly between the two groups (22 [50.0\%] vs. 20 [57.1\%], $p=1.00$ ). There was no significant difference in cervical lordosis between the AV and SF groups at each follow-up period (Table 2).

Pseudarthrosis most commonly occurred in the lowermost level in the AV (11/44, 25.0\%) and SF groups $(10 / 35,28.6 \%)$. Subsidence also most commonly occurred in the lowermost level in the AV (13/44, $29.5 \%)$ and SF groups $(11 / 35,31.4 \%)$. The distribution of the location of pseudarthrosis $(p=0.93)$ or subsidence $(p=0.29)$ did not significantly differ between the two groups (Table 2$)$.

At the proximal adjacent level, 3 (6.8\%) ALODs and 2 (4.5\%) ASDs were detected in the AV group, whereas $3(8.6 \%)$ ALODs and 2 (5.7\%) ASDs were identified in the SF group. The rates of ALOD $(p=1.00)$ and ASD $(p=1.00)$ did not significantly differ between the two groups. Plate-adjacent disc space distance did not significantly differ between the AV and SF groups ( $5.5 \pm 1.8$. vs. $5.3 \pm 1.8 \mathrm{~mm}, \mathrm{p}=0.60)$. Furthermore, the rate of plate-adjacent disc distance $<5 \mathrm{~mm}$ did not significantly differ between the two groups (14 [31.8\%] vs. 12 [34.3\%], $p=1.00$ ) (Table 3).

In the distal adjacent level, 3 (6.8\%) ALODs were noted in the AV group, and 2 (5.7\%) ALODs in the SF group $(p=1.00)$. There were no cases of ASD at the distal adjacent level in both groups. Plate-adjacent disc space distance did not significantly differ between the AV and SF groups $(7.4 \pm 2.3 \mathrm{~mm}$ vs $6.8 \pm$ $2.4 \mathrm{~mm}, \mathrm{p}=0.24)$. The rate of plate-adjacent disc distance $<5 \mathrm{~nm}$ also did not differ significantly between the two groups (7 [15.9\%] vs. $10[28.5 \%], p=0.27$ ) (Table 3 ).

The neck and arm pain VAS and NDI scores significantly improved at the final follow-up in both groups. Neck $(1.8 \pm 1.2$ vs $2.0 \pm 1.7, p=0.59)$ and arm pain VAS $(2.7 \pm 1.1$ vs $3.7 \pm 3.6, p=0.15)$ and NDI $(5.6 \pm 4.5$ vs $7.9 \pm 5.9, p=0.08)$ scores at the final follow-up did not significantly differ between the AV and SF groups (Table 4).

\section{Factors associated with pseudarthrosis}


A logistic regression analysis demonstrated that the number of levels operated was significantly associated with the occurrence of pseudarthrosis $(p=0.01)$ (Table 5$)$.

\section{Discussion}

Many previous studies have been performed to determine the optimal plating method for ACDF.2,10,12,13,22 One issue regarding the safe plating method is the decreasing incidence of ALOD. Lee et al. reported that the plate-disc space distance should be $>5 \mathrm{~mm}$ to decrease ALOD incidence. ${ }^{10}$ This technique involves inserting cranial and caudal screws from the corners immediately adjacent to their respective operativelevel end plate and placing the shortest plate that fits this screw placement. ${ }^{10,11}$ This technique, by limiting anterior longitudinal ligament dissection, is reported to decrease ALOD incidence with no additional complications. ${ }^{10,11}$

Other points to consider in anterior cervical plating are decreasing the amount of subsidence and the rate of pseudarthrosis. Park et al. reported that a short plate with an oblique screw trajectory construct is effective for decreasing the incidence of ALOD and subsidence. ${ }^{12} \mathrm{~A}$ screw length greater than $75 \%$ of the antero-posterior vertebral body diameter is recommended to decrease the pseudarthrosis rate. ${ }^{13}$ However, result comparisons between using the fixed and variable screws remain scarce.

The ALOD more commonly occurs at the proximal adjacent segment, and a plate-adjacent distance of < $5 \mathrm{~mm}$ is known as a risk factor. ${ }^{9,10}$ The ALOD limits motion at the adjacent segment and accelerates the degeneration of the level next to the adjacent level. ${ }^{11}$ This justifies the need to increase the plate-disc space distance at the cranial level. In contrast, pseudarthrosis or implant failure most commonly occurs at the caudal level. $6,15,16$ This can be explained by increased shear stress at the level near the cervicothoracic junction. ${ }^{23}$ However, the ALOD risk is relatively low at the distal adjacent segment, which emphasizes the need for additional stability at the caudal level, whereas there is less need to increase plate-disc space distance. ${ }^{9,10}$ Based on these previous findings, we attempted a hybrid construct using fixed screws selectively at the caudal level to increase its stability while inserting the variable screws at the cranial level.

The results of this study demonstrated that pseudarthrosis and subsidence most commonly occur at the caudal level. These results are consistent with the results of previous reports. ${ }^{6,15,16}$ However, the fusion rates of selective fixed constructs and all variable constructs did not differ significantly. Although the locking mechanism at the screw-plate interface of the fixed screw was expected to increase the stability of the caudal segment, it did not lead to increased fusion rate. The ALOD and ASD rates were not significantly different between the SF and AV groups. The cranial and caudal plate-adjacent disc space distance and rate of patients with a plate-adjacent disc space distance of $<5 \mathrm{~mm}$, which is a risk factor of ALOD, did not significantly differ between the two groups. Furthermore, clinical results, such as neck and arm pain VAS and NDI scores, did not significantly differ between the two groups. 
Since the rates of pseudarthrosis, subsidence, ALOD, and ASD were similar in both groups, the selective fixed screw construct did not seem to provide additional advantage over the all variable screw constructs. The variable screws are more advantageous than the fixed screws at the point where the insertion angle is freely modifiable and a longer screw can be inserted with increased angle. In contrast, the advantage of the fixed screw is that it can be easily inserted with constant angulation. Based on the results of this study, screw types can be selected based on individual patient's anatomy and surgeon's experience, without concern for increased pseudarthrosis or subsidence caused by screw type. Oh et al. also reported that fusion rates of using the fixed and variable screws are similar. This is consistent with the results of this study. ${ }^{22}$

Further studies should be conducted to clarify the method to decrease the rate of pseudarthrosis at the caudal level, especially for multi-level surgery, since the results of the current study did not demonstrate significant results by screw construct difference. Although Lu et al. reported that pseudarthrosis at the caudal level can be decreased by selectively using low-dose bone morphogenic protein at the caudal level, there is still concern regarding complications caused by bone morphogenic protein for anterior cervical surgery. ${ }^{16}$

Previous studies have demonstrated that the number of fusion levels, bone graft type, plating, sex, age, smoking, greater preoperative segmental motion, and greater $\mathrm{T} 1$ sagittal slopes are related factors associated with pseudarthrosis after ACDF. ${ }^{24-27}$ The result of the logistic regression analysis in this study also demonstrates that multi-level operation is a risk factor of pseudarthrosis, consistent with the results of previous studies. With increased fusion level, micromotion and contract stress also would have increased at the graft-bone interface, which could lead to pseudarthrosis. ${ }^{28}$

Our study has some limitations. First, this study had a limited sample size to assess the rate of ALOD or ASD. Second, there was a temporal difference in the type of operation performed. Although all operations were performed by a single surgeon at a single institute, unidentifiable factors due to time difference could have affected the results. Third, insertion angles and lengths of the screw were not considered as factors. However, a previous study demonstrated that screw insertion angle does not affect subsidence or fusion rate. ${ }^{22}$ Finally, this study has a potential bias due to the retrospective nature of this study.

In conclusion, the fusion rates, subsidence, patient-reported outcome measurements, plate-adjacent disc space distance, ALOD, and ASD were not significantly different between the selective caudal fixed screw and all variable screw constructs. The stability provided by the locking mechanism of the fixed screw did not lead to an increased fusion rate. Therefore, it would be better to select screws based on individual patient's anatomy and surgeon's experience without concern for increased complications caused by screw type.

\section{Declarations}

Data availability 
The datasets generated during and/or analyzed during the current study are available from the corresponding author on reasonable request.

\section{Acknowledgements}

This work was supported by the research program of Dongguk University.

\section{Author contributions}

Conceptualization and design of the work was done by JJY. Analysis, writing, and editing was done by SHP. Data collection was done by SYP.

\section{Competing interests}

The authors declare no competing interests.

\section{References}

1. Wang, M. et al. Anterior cervical discectomy and fusion performed using structural allograft or polyetheretherketone: pseudarthrosis and revision surgery rates with minimum 2-year follow-up. $J$ Neurosurg Spine, 1-8, doi:10.3171/2019.9.SPINE19879 (2019).

2. Hong, S. W. et al. A Comparison of fixed-hole and slotted-hole dynamic plates for anterior cervical discectomy and fusion. J Spinal Disord Tech23, 22-26, doi:10.1097/BSD.0b013e31819877e7 (2010).

3. Song, K. J., Taghavi, C. E., Lee, K. B., Song, J. H. \& Eun, J. P. The efficacy of plate construct augmentation versus cage alone in anterior cervical fusion. Spine (Phila Pa 1976)34, 2886-2892, doi:10.1097/BRS.0b013e3181b64f2c (2009).

4. Choi, S. H. \& Kang, C. N. Degenerative Cervical Myelopathy: Pathophysiology and Current Treatment Strategies. Asian Spine J14, 710-720, doi:10.31616/asj.2020.0490 (2020).

5. Alijani, B. \& Rasoulian, J. The Sagittal Balance of the Cervical Spine: Radiographic Analysis of Interdependence between the Occipitocervical and Spinopelvic Alignment. Asian Spine J14, 287-297, doi:10.31616/asj.2019.0165 (2020).

6. Park, S. et al. How Does Screw Migration or Fracture After Anterior Cervical Plate Fixation Affect the Radiographic and Clinical Outcomes? Clin Spine Surg32, 398-402, doi:10.1097/BSD.0000000000000844 (2019).

7. Song, K. J., Choi, B. W., Ham, D. H. \& Kim, H. J. Prognosis of Hardware-Related Problems in Anterior Cervical Discectomy and Fusion with Cage and Plate Constructs. World Neurosurg134, e249-e255, doi:10.1016/j.wneu.2019.10.042 (2020).

8. Yue, W. M., Brodner, W. \& Highland, T. R. Persistent swallowing and voice problems after anterior cervical discectomy and fusion with allograft and plating: a 5- to 11-year follow-up study. Eur Spine J14, 677-682, doi:10.1007/s00586-004-0849-3 (2005). 
9. Park, J. B., Cho, Y. S. \& Riew, K. D. Development of adjacent-level ossification in patients with an anterior cervical plate. J Bone Joint Surg Am87, 558-563, doi:10.2106/JBJS.C.01555 (2005).

10. Lee, D. H. et al. Anterior cervical plating technique to prevent adjacent-level ossification development. Spine J13, 823-829, doi:10.1016/j.spinee.2013.03.009 (2013).

11. Lee, D. H. et al. Adjacent Level Ossification Development Following Anterior Cervical Fusion: How Does it Affect the Next Level Disc? Spine (Phila Pa 1976)45, E1469-E1475, doi:10.1097/BRS.0000000000003656 (2020).

12. Park, J. H. et al. Efficacy of a Short Plate With an Oblique Screw Trajectory for Anterior Cervical Plating: A Comparative Study With a 2-Year Minimum Follow-Up. Clin Spine Surg29, E43-48, doi:10.1097/BSD.0000000000000111 (2016).

13. Lee, N. J. et al. Does screw length for primary two-level ACDF influence pseudarthrosis risk? Spine J20, 1752-1760, doi:10.1016/j.spinee.2020.07.002 (2020).

14. Rhee, J. M. \& Riew, K. D. Dynamic anterior cervical plates. J Am Acad Orthop Surg15, 640-646, doi:10.5435/00124635-200711000-00002 (2007).

15. Wewel, J. T. et al. Fusion rate following three- and four-level ACDF using allograft and segmental instrumentation: A radiographic study. J Clin Neurosci62, 142-146, doi:10.1016/j.jocn.2018.11.040 (2019).

16. Lu, D. C., Tumialan, L. M. \& Chou, D. Multilevel anterior cervical discectomy and fusion with and without rhBMP-2: a comparison of dysphagia rates and outcomes in 150 patients. J Neurosurg Spine18, 43-49, doi:10.3171/2012.10.SPINE10231 (2013).

17. Marques, C. et al. Accuracy and Reliability of X-ray Measurements in the Cervical Spine. Asian Spine J14, 169-176, doi:10.31616/asj.2019.0069 (2020).

18. Song, K. S. et al. Dynamic radiographic criteria for detecting pseudarthrosis following anterior cervical arthrodesis. J Bone Joint Surg Am96, 557-563, doi:10.2106/JBJS.M.00167 (2014).

19. Song, K. S. et al. Anterior cervical fusion assessment using reconstructed computed tomographic scans: surgical confirmation of 254 segments. Spine (Phila Pa 1976)38, 2171-2177, doi:10.1097/BRS.0000000000000017 (2013).

20. Goffin, J. et al. Long-term follow-up after interbody fusion of the cervical spine. J Spinal Disord Tech17, 79-85, doi:10.1097/00024720-200404000-00001 (2004).

21. Park, S. et al. Feasibility of local bone dust as a graft material in anterior cervical discectomy and fusion. J Neurosurg Spine, 1-6, doi:10.3171/2019.3.SPINE181416 (2019).

22. Oh, K., Lee, C. K., You, N. K., Kim, S. H. \& Cho, K. H. Radiologic changes of anterior cervical discectomy and fusion using allograft and plate augmentation: comparison of using fixed and variable type screw. Korean J Spine10, 160-164, doi:10.14245/kjs.2013.10.3.160 (2013).

23. Smith, G. A. et al. Incidence and Outcomes of Acute Implant Extrusion Following Anterior Cervical Spine Surgery. Global Spine J, 40S-45S, doi:10.1177/2192568216686752 (2017). 
24. Wang, J. C., McDonough, P. W., Endow, K. K. \& Delamarter, R. B. Increased fusion rates with cervical plating for two-level anterior cervical discectomy and fusion. Spine (Phila Pa 1976)25, 41-45, doi:10.1097/00007632-200001010-00009 (2000).

25. Samartzis, D. et al. Comparison of allograft to autograft in multilevel anterior cervical discectomy and fusion with rigid plate fixation. Spine J3, 451-459, doi:10.1016/s1529-9430(03)00173-6 (2003).

26. Shen, H. X. et al. Pseudarthrosis in multilevel anterior cervical fusion with rhBMP-2 and allograft: analysis of one hundred twenty-seven cases with minimum two-year follow-up. Spine (Phila Pa 1976)35, 747-753, doi:10.1097/BRS.0b013e3181bc3420 (2010).

27. Choi, S. H. et al. Preoperative Radiographic Parameters to Predict a Higher Pseudarthrosis Rate After Anterior Cervical Discectomy and Fusion. Spine (Phila Pa 1976)42, 1772-1778, doi:10.1097/BRS.0000000000002219 (2017).

28. Lee, D. H. et al. What Is the Fate of Pseudarthrosis Detected 1 Year After Anterior Cervical Discectomy and Fusion? Spine (Phila Pa 1976)43, E23-E28, doi:10.1097/BRS.0000000000002077 (2018).

\section{Tables}

\section{Table 1. Patient characteristics}




\begin{tabular}{|c|c|c|c|}
\hline & AV group & SF group & $P$ value \\
\hline Age & $56.1 \pm 12.4$ & $56.1 \pm 12.2$ & 0.99 \\
\hline \multicolumn{3}{|l|}{ Sex } & \multirow[t]{3}{*}{1.00} \\
\hline Male & $26(59.1 \%)$ & $21(60.0 \%)$ & \\
\hline Female & $18(40.9 \%)$ & $14(40.0 \%)$ & \\
\hline \multicolumn{3}{|l|}{ Diagnosis } & \multirow[t]{3}{*}{$0.02^{*}$} \\
\hline Radiculopathy & 35 (79.5\%) & $18(51.4 \%)$ & \\
\hline Myelopathy & $9(20.5 \%)$ & $17(48.6 \%)$ & \\
\hline Smoking status & $10(22.3 \%)$ & $11(31.4 \%)$ & 0.45 \\
\hline BMI $\left(\mathrm{kg} / \mathrm{m}^{2}\right)$ & $26.1 \pm 3.9$ & $24.7 \pm 5.4$ & 0.17 \\
\hline $\operatorname{BMD}\left(\mathrm{g} / \mathrm{cm}^{2}\right)$ & $1.0 \pm 0.2$ & $0.9 \pm 0.2$ & 0.23 \\
\hline Follow-up period (m) & $49.8 \pm 14.9$ & $47.6 \pm 30.6$ & 0.68 \\
\hline Number of levels & $1.6 \pm 0.7$ & $1.7 \pm 0.7$ & 0.75 \\
\hline \multicolumn{4}{|l|}{ Complications } \\
\hline Dural tear & $0(0.0 \%)$ & $0(0.0 \%)$ & $n / a$ \\
\hline Hematoma & $0(0.0 \%)$ & $0(0.0 \%)$ & $\mathrm{n} / \mathrm{a}$ \\
\hline Infection & $1(2.3 \%)$ & $0(0.0 \%)$ & 1.00 \\
\hline Readmission & $0(0.0 \%)$ & $1(2.9 \%)$ & 0.44 \\
\hline Reoperation & $1(2.3 \%)$ & $1(2.9 \%)$ & 1.00 \\
\hline Neurologic deficit & $0(0.0 \%)$ & $0(0.0 \%)$ & $\mathrm{n} / \mathrm{a}$ \\
\hline \multicolumn{4}{|c|}{ AV, all variable; SF, selective caudal; BMI, body mass index; $m$, months; $n / a$, not availabl } \\
\hline \multicolumn{4}{|c|}{ Age, BMI, follow-up period, number of levels were analyzed using a student's t-test } \\
\hline \multicolumn{4}{|c|}{ Sex, diagnosis, smoking status, complications were analyzed using a chi-square test } \\
\hline * $P<0.05$ & & & \\
\hline
\end{tabular}

\section{Table 2. Radiographic results}




\begin{tabular}{|c|c|c|c|}
\hline & AV group & SF group & $P$ value \\
\hline Fusion CT & $28(63.6 \%)$ & $20(57.1 \%)$ & 0.64 \\
\hline Fusion ISM & $30(68.1 \%)$ & $26(74.3 \%)$ & 0.38 \\
\hline Subsidence & $26(59.1 \%)$ & $20(57.1 \%)$ & 1.00 \\
\hline \multicolumn{4}{|l|}{ C2-C7 lordosis } \\
\hline Preoperative & $12.6 \pm 10.1$ & $14.9 \pm 10.5$ & 0.34 \\
\hline Postoperative & $17.5 \pm 9.8$ & $20.2 \pm 8.6$ & 0.20 \\
\hline Final follow-up & $16.0 \pm 9.8$ & $17.9 \pm 7.9$ & 0.36 \\
\hline \multicolumn{3}{|l|}{ Pseudarthrosis location } & \multirow[t]{6}{*}{0.93} \\
\hline Single level operation & $4(9.1 \%)$ & $4(11.4 \%)$ & \\
\hline \multicolumn{3}{|l|}{ Multi-level operation } & \\
\hline Lowermost & $11(25.0 \%)$ & $10(28.6 \%)$ & \\
\hline Other level & $2(4.5 \%)$ & $3(8.6 \%)$ & \\
\hline Multiple locations & $1(2.3 \%)$ & $2(5.7 \%)$ & \\
\hline Subsidence location & & & \multirow[t]{6}{*}{0.29} \\
\hline Single level operation & $13(29.5 \%)$ & $7(20.0 \%)$ & \\
\hline \multicolumn{3}{|l|}{ Multi-level operation } & \\
\hline Lowermost & $13(29.5 \%)$ & $11(31.4 \%)$ & \\
\hline Other level & $9(20.5 \%)$ & $6(17.1 \%)$ & \\
\hline Multiple locations & $9(20.5 \%)$ & $4(11.4 \%)$ & \\
\hline \multicolumn{4}{|c|}{ AV, all variable; SF, selective fixed; ISM, interspinous motion; } \\
\hline \multicolumn{4}{|c|}{$\begin{array}{l}\text { Fusion, subsidence, location of pseudarthrosis, and location of subsidence were analyzed using a chi- } \\
\text { square test }\end{array}$} \\
\hline \multicolumn{4}{|c|}{ C2-C7 lordosis was analyzed using a student's t-test } \\
\hline$\star P<0.05$ & & & \\
\hline
\end{tabular}

\section{Table 3. Radiographic results regarding ALOD and ASD}




\begin{tabular}{|c|c|c|c|}
\hline & AV group & SF group & $P$ value \\
\hline \multicolumn{4}{|l|}{ Proximal adjacent level } \\
\hline ALOD & $3(6.8 \%)$ & $3(8.6 \%)$ & 1.00 \\
\hline ASD & $2(4.5 \%)$ & $2(5.7 \%)$ & 1.00 \\
\hline Plate-adjacent disc distance $(\mathrm{mm})$ & $5.5 \pm 1.8$ & $5.3 \pm 1.8$ & 0.60 \\
\hline Plate-adjacent disc distance $<5 \mathrm{~mm}$ & $14(31.8 \%)$ & $12(34.3 \%)$ & 1.00 \\
\hline \multicolumn{4}{|l|}{ Distal adjacent level } \\
\hline ALOD & $3(6.8 \%)$ & $2(5.7 \%)$ & 1.00 \\
\hline ASD & $0(0.0 \%)$ & $0(0.0 \%)$ & $\mathrm{n} / \mathrm{a}$ \\
\hline Plate-adjacent disc distance & $7.4 \pm 2.3$ & $6.8 \pm 2.4$ & 0.24 \\
\hline Plate-adjacent disc distance $<5 \mathrm{~mm}$ & $7(15.9 \%)$ & $10(28.5 \%)$ & 0.27 \\
\hline \multicolumn{4}{|c|}{$\begin{array}{l}\text { AV, all variable; SF, selective fixed; ALOD, adjacent level ossification development; ASD, adjacent } \\
\text { segmental disease; n/a, not available }\end{array}$} \\
\hline \multicolumn{4}{|c|}{ ALOD, ASD, and plate-adjacent disc distance $<5 \mathrm{~mm}$ were analyzed using a chi-square test; } \\
\hline Plate-adjacent disc distance was ana & udent's t-tes & & \\
\hline
\end{tabular}


Table 4

Patient reported outcome measure results

\begin{tabular}{|c|c|c|c|c|}
\hline & & AV group & SF group & $P$ valuet \\
\hline \multirow{3}{*}{$\begin{array}{l}\text { Neck pain } \\
\text { VAS }\end{array}$} & Preoperative & $7.5 \pm 1.4$ & $7.1 \pm 2.7$ & 0.33 \\
\hline & Final & $1.8 \pm 1.2$ & $2.0 \pm 1.7$ & 0.59 \\
\hline & P value $\neq$ & $<0.01^{*}$ & $<0.01^{*}$ & \\
\hline \multirow{3}{*}{$\begin{array}{l}\text { Arm pain } \\
\text { VAS }\end{array}$} & Preoperative & $7.8 \pm 1.6$ & $8.1 \pm 1.5$ & 0.62 \\
\hline & Final & $2.7 \pm 1.1$ & $3.7 \pm 3.6$ & 0.15 \\
\hline & $P$ value $\ddagger$ & $<0.01^{*}$ & $<0.01^{*}$ & \\
\hline \multirow[t]{3}{*}{ NDI } & Preoperative & $18.6 \pm 4.8$ & $23.6 \pm 6.4$ & $<0.01^{*}$ \\
\hline & Final & $5.6 \pm 4.5$ & $7.9 \pm 5.9$ & 0.08 \\
\hline & P value $\ddagger$ & $<0.01^{\star}$ & $<0.01^{\star}$ & \\
\hline \multicolumn{5}{|c|}{ AV, all variable; SF, selective fixed; VAS, visual analogue scale; NDI, neck disability index; } \\
\hline \multicolumn{5}{|c|}{ † Comparison between two groups were performed by student's t-test } \\
\hline \multicolumn{5}{|c|}{ ‡ Comparison between preoperative and postoperative values were performed by paired t-test } \\
\hline \multicolumn{5}{|l|}{$\star P<0.05$} \\
\hline
\end{tabular}

Table 5

Logistic regression analysis demonstrating factors associated with nonunion

\begin{tabular}{|llll|}
\hline Univariate analysis & Odds ratio & Confidence interval & P value \\
\hline Age & 0.99 & $0.951-1.025$ & 0.50 \\
\hline Number of levels operated & 2.52 & $1.224-5.175$ & 0.01 * \\
\hline Smoking status & 0.82 & $0.296-2.246$ & 0.69 \\
\hline BMI & 1.10 & $0.977-1.240$ & 0.11 \\
\hline BMD & 0.95 & $0.774-1.175$ & 0.66 \\
\hline Screw construct type & 0.76 & $0.307-1.890$ & 0.56 \\
\hline Subsidence & 0.52 & $0.202-1.328$ & 0.17 \\
\hline Preoperative lordosis & 0.99 & $0.946-1.034$ & 0.63 \\
\hline BMI, body mass index; BMD, bone mineral density; & \\
\hline * P<0.05 & & & \\
\hline
\end{tabular}




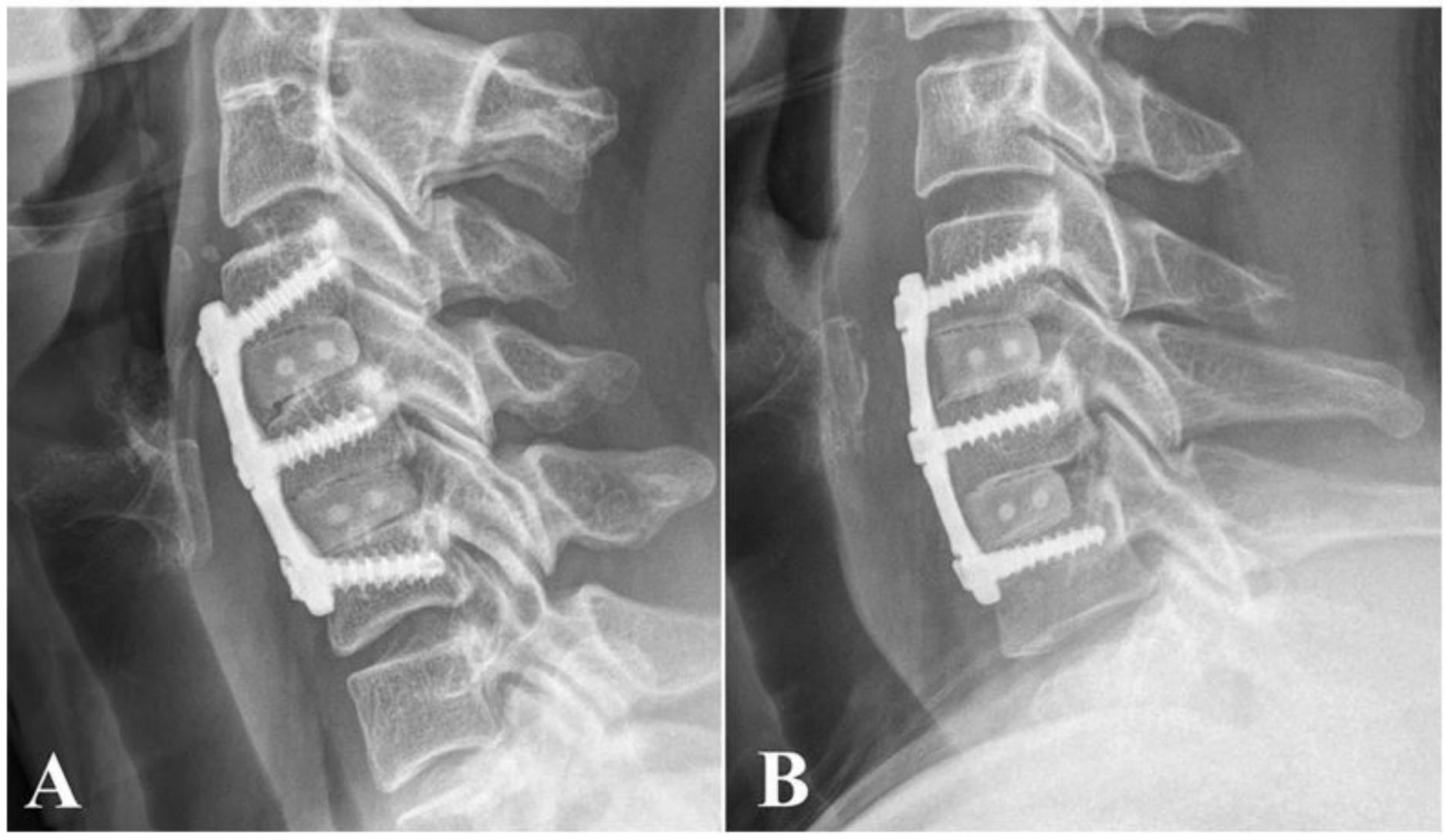

\section{Figure 1}

All variable screw construct and selective caudal fixed screw construct (A) All variable screw construct. A short plate was selected to maintain the plate-adjacent disc space distance at $>5 \mathrm{~mm}$. The screw was directed obliquely to insert the longest screw possible. (B) Selective caudal fixed screw construct. Fixed screws were inserted at the caudal level to increase stability. Variable screws were inserted at the cranial level and middle level. Fixed screw insertion at the caudal level allowed less caudal angulation. 


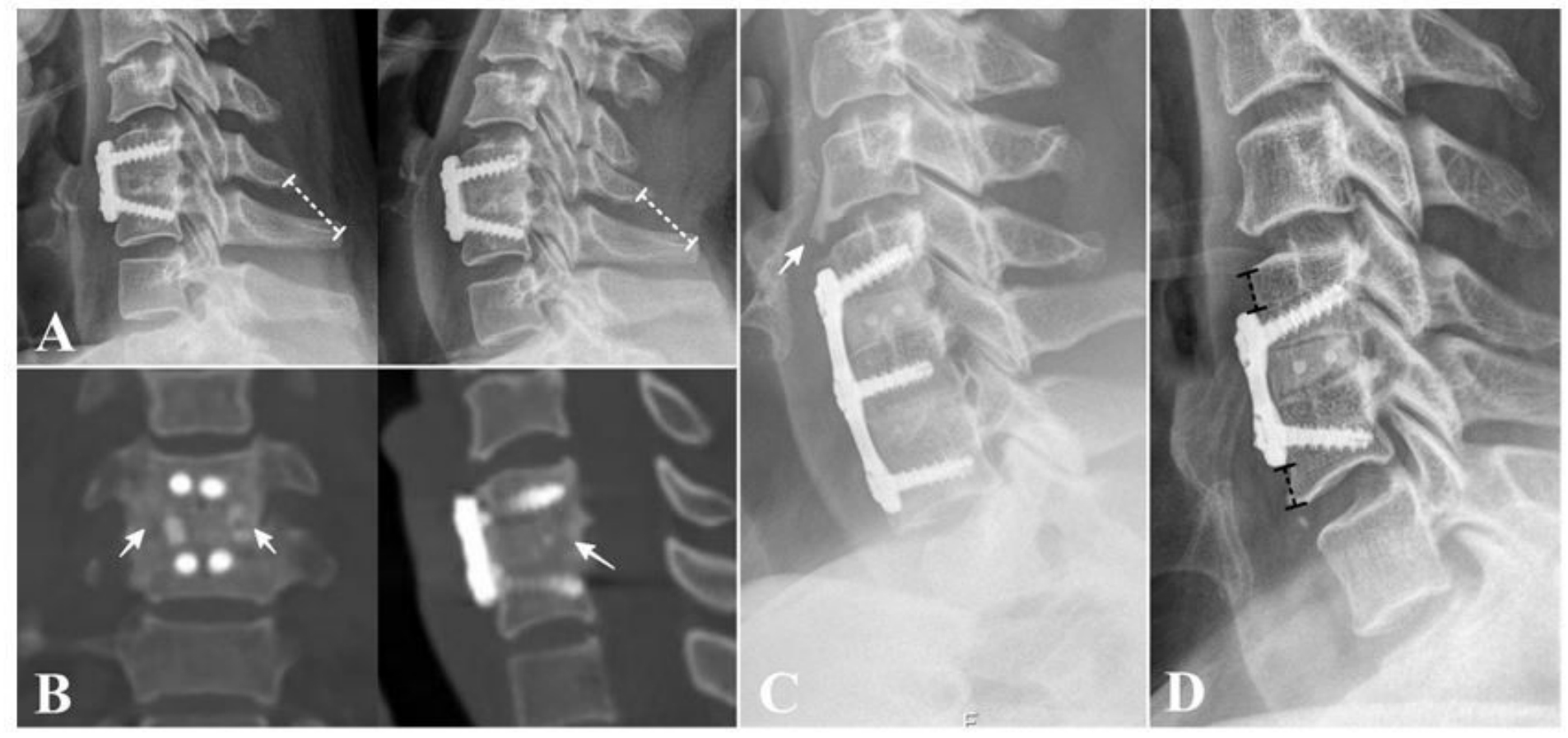

\section{Figure 2}

Radiographic measurements (A) Fusion by interspinous motion. Interspinous motion $<2 \mathrm{~mm}$ on $150 \%$ magnified flexion/extension lateral radiograph was considered as fusion. (B) Fusion was achieved by bone bridging. Bone bridging formation demonstrated both on coronal and sagittal reconstructed CT images were assessed as fusion. (C) Ossification of the anterior longitudinal ligament crossing the adjacent disc space demonstrated on lateral cervical radiograph was considered as an adjacent level ossification development. (D) Plate-adjacent disc space distance. The distance between the tips of the plate to the cephalad and caudal adjacent disc spaces on the immediate postoperative lateral radiograph of the cervical spine is shown. 